\title{
Cutaneous soft tissue tumors: how do we make sense of fibrous and "fibrohistiocytic" tumors with confusing names and similar appearances?
}

\author{
Jason L. Hornick $\mathbb{D}^{1}$
}

Received: 31 August 2019 / Revised: 1 October 2019 / Accepted: 1 October 2019 / Published online: 25 October 2019

(c) The Author(s), under exclusive licence to United States \& Canadian Academy of Pathology 2019

\begin{abstract}
In the 2018 World Health Organization Classification of Skin Tumors, a wide range of predominantly benign mesenchymal neoplasms are included in the fibroblastic, myofibroblastic, and "fibrohistiocytic" categories. By far the most common of these tumors is dermatofibroma (fibrous histiocytoma). There are many histologic variants of dermatofibroma, some of which (cellular, aneurysmal, and atypical) are associated with a higher risk of local recurrence; these variants may be mistaken for more aggressive tumor types, including sarcomas. Furthermore, distinguishing among the fibrous and "fibrohistiocytic" tumors can be a diagnostic challenge, given their sometimes-similar histologic appearances and confusing nomenclature. Immunohistochemistry and molecular genetic assays play a relatively limited role in the diagnosis of these tumor types, with notable exceptions (i.e., epithelioid fibrous histiocytoma and dermatofibrosarcoma protuberans). Proper recognition of dermatofibrosarcoma protuberans is critical, since this tumor type is associated with locally aggressive behavior; transformation to the fibrosarcomatous variant brings metastatic potential. In recent years, understanding of the molecular pathogenetic basis for cutaneous mesenchymal neoplasms has increased dramatically, with the discovery of gene rearrangements in some of these tumor types. In this review, the histologic features of the most common fibrous and "fibrohistiocytic" cutaneous mesenchymal neoplasms will be discussed, as well as recently identified molecular genetic alterations.
\end{abstract}

\section{Introduction}

The concept of "fibrohistiocytic" tumors was established around 60 years ago, when the term "malignant fibrous histiocytoma" was applied to pleomorphic sarcomas, based on morphologic appearances (i.e., tumor cells resemble both fibroblasts and histiocytes) and observed behavior in tissue culture, in which the tumor cells showed ameboid growth and phagocytic properties and were believed to represent "facultative fibroblasts" that were histiocytic in origin [1]. The term "fibrous histiocytoma" was subsequently applied to benign and intermediate neoplasms of skin and superficial soft tissue. However, neither benign nor malignant lesions in these categories are in fact derived from histiocytes (they are

Jason L. Hornick

jhornick@bwh.harvard.edu

1 Department of Pathology, Brigham and Women's Hospital, Harvard Medical School, Boston, MA, United States mesenchymal in nature). "Malignant fibrous histiocytoma" is now known to have represented a wastebasket category that included distinct and heterogeneous sarcoma types (as well as some sarcomatoid carcinomas and melanomas), which can be distinguished using a combination of histologic features, immunohistochemistry, and molecular genetics; subclassification of pleomorphic sarcomas has prognostic significance, given marked differences in metastatic potential [2-4]. The term "dermatofibroma" is a more appropriate designation than "fibrous histiocytoma" (since the latter term is in fact meaningless) and will be used in this review.

In the 2018 World Health Organization Classification of Skin Tumors, a wide range of predominantly benign mesenchymal neoplasms are included in the fibroblastic, myofibroblastic, and "fibrohistiocytic" categories (see Table 1) [5]. By far the most common of these tumors is dermatofibroma. There are many histologic variants of dermatofibroma, some of which (cellular, aneurysmal, and atypical) are associated with a higher risk of local recurrence (up to 20\%); these tumor types may be mistaken for sarcomas. Furthermore, distinguishing among cutaneous fibrous and "fibrohistiocytic" 
Table 12018 World Health Organization Classification of Skin Tumors

Fibroblastic, myofibroblastic, and fibrohistiocytic tumors

\begin{tabular}{ll}
\hline $\begin{array}{l}\text { Dermatofibroma (fibrous } \\
\text { histiocytoma) } \\
\text { Epithelioid fibrous histiocytoma }\end{array}$ & $\begin{array}{l}\text { Dermatomyofibroma } \\
\text { flexiform } \\
\text { fibrohistiocytic tumor } \\
\text { Plaque-like CD34+ dermal } \\
\text { fibroma }\end{array}$ \\
$\begin{array}{l}\text { Fibrosarcomatous } \\
\text { dermatofibrosarcoma protuberans }\end{array}$ & $\begin{array}{l}\text { Fibromas (8 distinct } \\
\text { tumor types) }\end{array}$ \\
Giant cell fibroblastoma & Myofibroma/myofibromatosis \\
Superficial fibromatosis & Nodular fasciitis \\
Superficial acral fibromyxoma & Cutaneous myxoma
\end{tabular}

tumors can be a diagnostic challenge, given their sometimessimilar histologic appearances and confusing nomenclature. Immunohistochemistry and molecular genetic assays play a relatively limited role in the diagnosis of these tumor types, with notable exceptions. Proper recognition of dermatofibrosarcoma protuberans is critical, since this tumor type is associated with locally aggressive behavior; transformation to the fibrosarcomatous variant brings metastatic potential. In recent years, understanding of the molecular pathogenetic basis for cutaneous mesenchymal neoplasms has increased dramatically, with the discovery of gene rearrangements in some of these tumor types (including dermatofibroma and variants and epithelioid fibrous histiocytoma). In this review, the histologic features of the most common cutaneous fibrous and "fibrohistiocytic" mesenchymal neoplasms will be discussed, as well as recently identified molecular genetic alterations.

\section{Dermatofibroma (benign fibrous histiocytoma) and variants}

Dermatofibroma is the most common mesenchymal tumor of skin. This tumor type arises over a wide age range (with a peak incidence in young to middle-aged adults) and anatomic distribution (with a predilection for the trunk and extremities). The dermatopathologist and surgical pathologist should always consider the possibility of dermatofibroma when encountering a cutaneous spindle cell neoplasm, especially as there are many histologic variants of this tumor type. Several of these variants are associated with an increased rate of local recurrence (see Table 2); moreover, some variants may be confused with dermatofibrosarcoma protuberans or sarcomas. It is therefore critical to diagnose such tumors accurately. Histologic features that can help identify dermatofibromas and variants include
Table 2 Recurrence rates for dermatofibroma (fibrous histiocytoma) and variants

\begin{tabular}{ll}
\hline Variant & Recurrence \\
\hline Common dermatofibroma & $<5 \%$ \\
Cellular dermatofibroma & $20 \%$ \\
Aneurysmal dermatofibroma & $20 \%$ \\
Atypical dermatofibroma & $20 \%$ \\
\hline
\end{tabular}

relative circumscription, overlying epidermal hyperplasia, and lateral entrapment of hyaline dermal collagen; these features should be sought when encountering a cutaneous spindle cell neoplasm. The cellular, aneurysmal, and atypical variants of dermatofibroma will be briefly discussed.

Cellular dermatofibromas typically show a variably fascicular and storiform architecture and are composed of uniform spindle cells with fine chromatin and pale eosinophilic cytoplasm (Fig. 1). In addition to the general features of dermatofibroma listed above, cellular dermatofibromas (and the other variants discussed below) often show entrapment of superficial subcutaneous fat; small foci of central necrosis are found in around $10 \%$ of cases [6]. These latter features in particular may raise concern for a more aggressive tumor type. Immunohistochemistry plays a relatively limited role in the diagnosis of cellular dermatofibroma and variants, although nearly all cases show multifocal staining for SMA (see Table 3) [7]. The immunophenotypic findings support myofibroblastic differentiation. Although immunohistochemistry for factor XIIIa is widely used in dermatopathology practice, in fact this marker is usually expressed in reactive dermal fibroblasts ("dendrocytes") that surround the tumor, less often in tumor cells; this author does not use factor XIIIa in his practice.

Aneurysmal dermatofibroma contains blood-filled clefts or pseudocystic spaces, leading to the common clinical suspicion for a hemangioma. Similar to other variants, overlying epidermal hyperplasia and lateral collagen entrapment are characteristic findings (Fig. 2). These tumors usually contain abundant hemosiderin and are polymorphous in composition, including, in addition to the neoplastic spindle cells, foam cells, hemosiderin-laden macrophages, lymphocytes, and often touton-type giant cells [8]. Aneurysmal fibrous histiocytoma should not be confused with angiomatoid fibrous histiocytoma (a term unfortunately used for aneurysmal dermatofibroma in some publications), which is an entirely unrelated tumor type. Angiomatoid fibrous histiocytoma is a neoplasm of uncertain differentiation that typically arises in the subcutis of the proximal extremities in children and young adults [9-11]. In addition to a potential for local recurrence (10-15\%), angiomatoid fibrous histiocytoma occasionally metastasizes 
Fig. 1 Cellular dermatofibroma. a The tumor is relatively wellcircumscribed with an expansile appearance. b There is overlying epidermal hyperplasia. c Lateral entrapment of hyaline dermal collagen is a characteristic finding. d The tumor shows a predominantly fascicular architecture and is composed of spindle cells with fine chromatin and pale eosinophilic cytoplasm
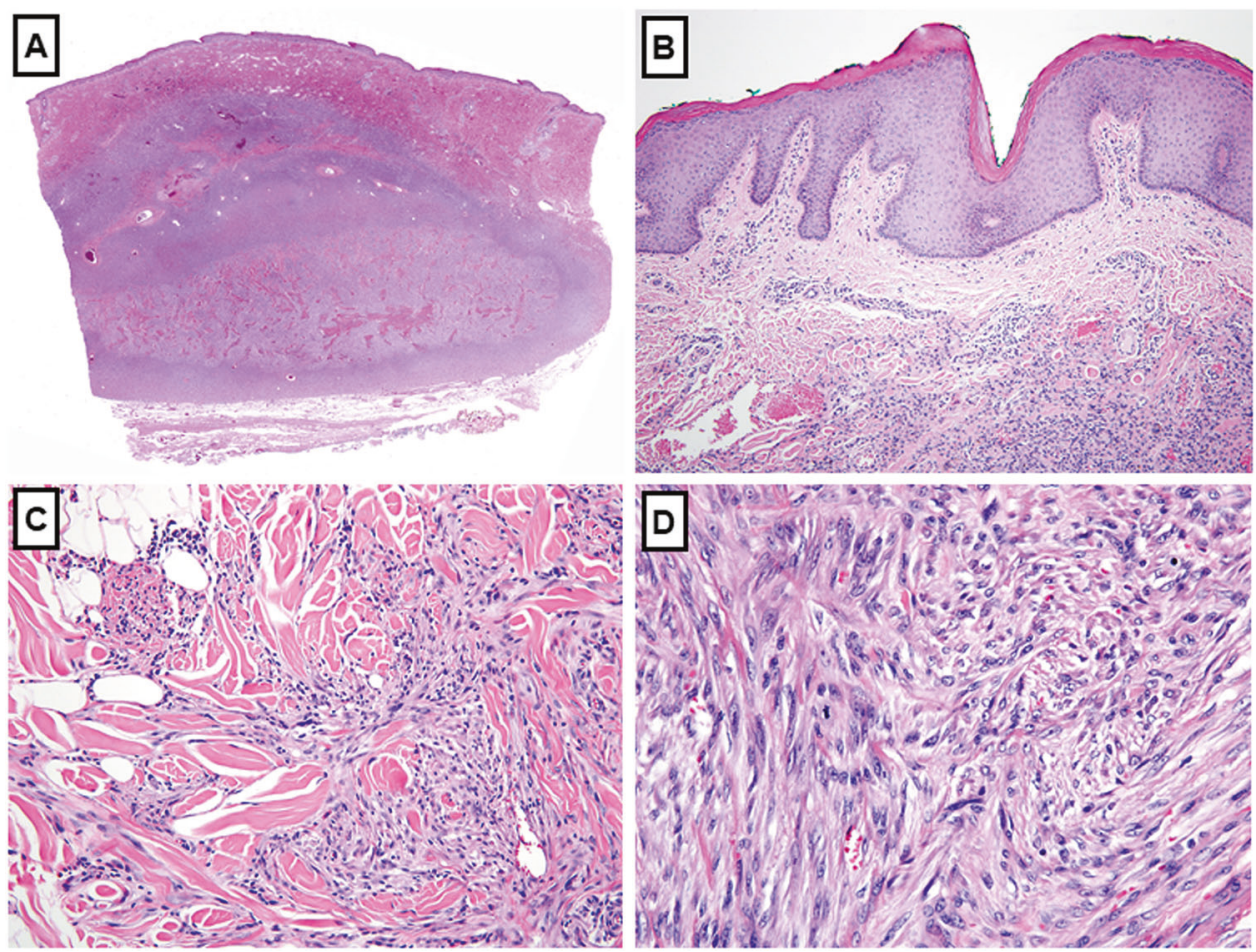

Table 3 Immunophenotypic findings in cellular dermatofibroma ${ }^{a}$

\begin{tabular}{lc}
\hline Marker (extent) & Positive \\
\hline SMA (multifocal) & $95 \%$ \\
Desmin (focal) & $30 \%$ \\
CD34 (focal) & $5 \%$ \\
\hline
\end{tabular}

${ }^{a}$ Factor XIIIa is usually negative in the lesional cells of cellular dermatofibroma but is expressed in surrounding dermal fibroblasts

to lymph nodes (in around 2\% of cases), and exceptionally rarely to the lungs and other sites with tumor-associated mortality [9-11]. Angiomatoid fibrous histiocytoma often contains dilated, pseudocystic spaces, a peripheral lymphoid cuff including germinal centers, and dense fibrosis; the lymphoid component may lead to the mistaken impression of a lymph node involved by a metastatic neoplasm. The tumor cells are often arranged in nodules within the walls of the pseudocysts and show histiocytoid, epithelioid, or spindle cell morphology with vesicular nuclei and abundant, syncytial eosinophilic cytoplasm. Some cases lack cystic spaces, lack a peripheral lymphoid cuff, or show morphologic variations including myxoid stroma or atypical/ pleomorphic cytomorphology. The unusual combination of desmin and EMA expression by immunohistochemistry is typical and can be a helpful diagnostic finding. Angiomatoid fibrous histiocytomas harbor characteristic gene fusions: either EWSR1-CREB1 (which predominates) or
EWSR1-ATF1 [12, 13], remarkably the same fusions found in clear cell sarcoma of tendons and aponeuroses, an entirely unrelated tumor type with melanocytic differentiation and aggressive metastatic behavior. Fluorescence in situ hybridization (FISH) for EWSRl can therefore be used to support the diagnosis of angiomatoid fibrous histiocytoma.

Atypical dermatofibroma, in addition to overlying epidermal hyperplasia and lateral entrapment of hyaline dermal collagen bundles similar to other dermatofibroma variants, contains scattered atypical or pleomorphic cells; mitotic activity may be seen, including atypical mitotic figures (Fig. 3) [14]. The remaining cells may be relatively uniform spindle cells similar to cellular dermatofibroma or polymorphous, similar to conventional dermatofibroma or other variants. Atypical dermatofibroma is most likely to be mistaken for a pleomorphic sarcoma; careful attention to the classic features of dermatofibroma from low magnification is therefore important to avoid misdiagnosis.

Recent studies have identified recurrent gene fusions in dermatofibromas (including the variants discussed in this review) involving protein kinase $\mathrm{C}$ isoforms (PRKCA, $P R K C B$, and $P R K C D)$ and various fusion partners, most encoding membrane-associated proteins (e.g., KIRREL, PDPN, CD63, and LAMTOR1) [15]. Although these discoveries have not been incorporated into clinical practice, they provide clear evidence for the neoplastic, molecular 
Fig. 2 Aneurysmal dermatofibroma. a The tumor contains blood-filled cystic spaces. b There is fine hemosiderin in foam cells admixed with tumor cells adjacent to the areas of hemorrhage. c Overlying epidermal hyperplasia is a typical finding. d The tumor extends into the superficial subcutaneous fat and shows e entrapment of lateral hyaline dermal collagen. $f$ The neoplastic spindle cells show a storiform growth pattern. Note the hemorrhage and touton-type giant cells
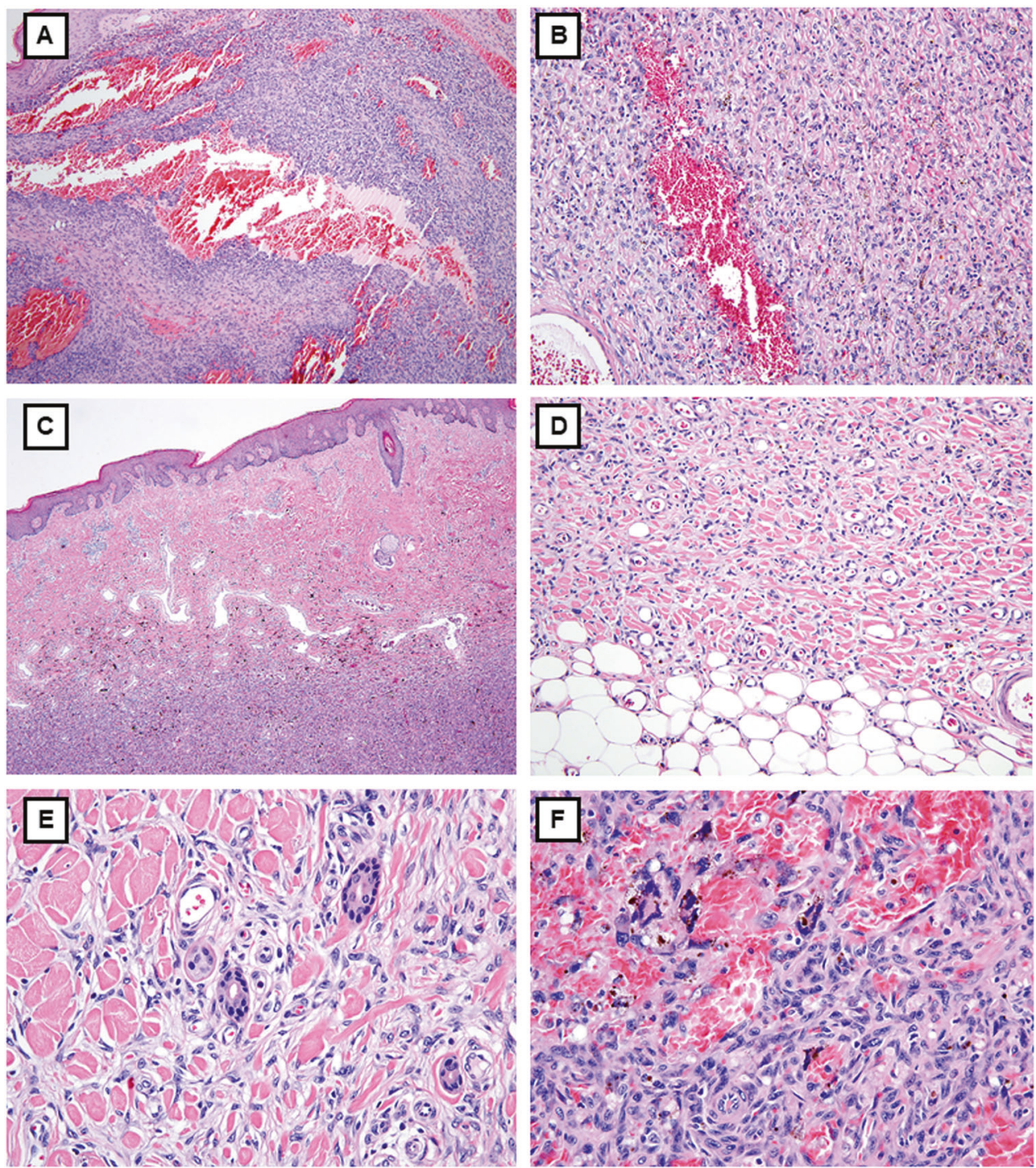

pathogenetic basis for dermatofibromas. Of note, these gene fusions are only found in a minority of cases [16]; it is likely that other gene rearrangements will be discovered in dermatofibromas in due course.

Epithelioid fibrous histiocytoma (also known as "epithelioid cell histiocytoma") has traditionally been considered to be a morphologic variant of cutaneous fibrous histiocytoma (dermatofibroma). Epithelioid fibrous histiocytoma arises on the extremities of young to middleaged adults and shows distinct features from conventional dermatofibroma and variants: an exophytic, wellcircumscribed appearance, sometimes with an epidermal collarette; uniform epithelioid cells with small nucleoli and eosinophilic or amphophilic cytoplasm, including occasional binucleated cells; often prominent small, rounded blood vessels; and a sheet-like or vaguely whorled architecture (Fig. 4) [17-19]. EMA is often positive in epithelioid fibrous histiocytoma [20]. These histologic differences have led investigators to suggest that epithelioid fibrous histiocytoma is not in fact related to conventional dermatofibroma (see Table 4). Furthermore, recent studies have identified consistent $A L K$ gene rearrangements in epithelioid fibrous histiocytoma, with a wide range of fusion partners (most often SQSTM1-ALK or VCL-ALK; strikingly, these gene fusions are also found in the rare ALK-positive diffuse large B-cell lymphoma and an aggressive renal cell carcinoma variant, respectively) [21-24]. These $A L K$ gene rearrangements result in highlevel expression of the ALK protein, which can be detected by immunohistochemistry (see Fig. 4d); we now have a simple tool to confirm the diagnosis of this rare tumor type [22]. Interesting, several ALK-negative tumors classified as epithelioid fibrous histiocytoma have been found to harbor $P R K C B$ rearrangements [16], suggesting that occasional tumors in fact represent conventional dermatofibromas with epithelioid morphology. 
Fig. 3 Atypical dermatofibroma. a Similar to other dermatofibroma variants, the tumor is relatively circumscribed and shows overlying epidermal hyperplasia. b The tumor shows a variably fascicular-to-storiform growth pattern. c Entrapment of hyaline dermal collagen is a helpful diagnostic clue. d The degree of nuclear atypia and pleomorphism can be striking. Without careful attention to the other histologic features, there is significant risk for misdiagnosis as sarcoma
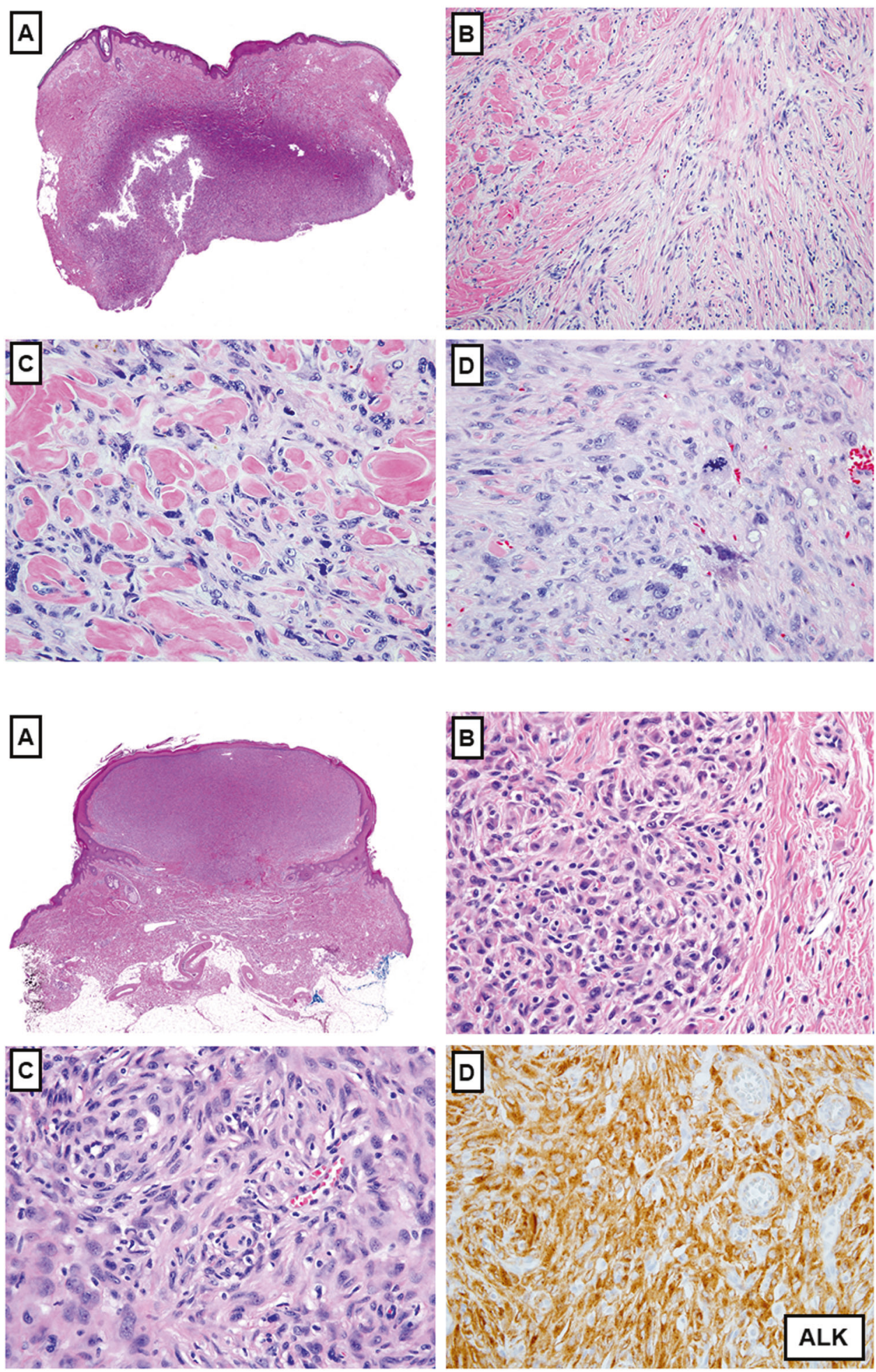

\section{Dermatofibrosarcoma protuberans and variants}

Although their names are similar, dermatofibrosarcoma protuberans has no relationship with dermatofibroma. Despite its name, dermatofibrosarcoma protuberans is not in fact a sarcoma; it is a mesenchymal neoplasm of intermediate biologic potential, locally aggressive [25]. Dermatofibrosarcoma protuberans does not have metastatic potential in its conventional form (which is required to classify a mesenchymal neoplasm as a sarcoma); only when transformation to the fibrosarcomatous variant occurs can 
Table 4 Is epithelioid fibrous histiocytoma related to conventional dermatofibromas?

\begin{tabular}{lll}
\hline Features & Dermatofibroma & Epithelioid fibrous histiocytoma \\
\hline Overlying epidermal hyperplasia & Present & Absent \\
Tumor margins & Lateral entrapment of dermal collagen bundles & Sharply circumscribed \\
Cytology & Short spindle cells & Epithelioid cells \\
Inflammatory infiltrate & Often prominent lymphocytes; sometimes foam cells & Variable lymphocytes \\
Gene fusions & $P R K C A, P R K C B, P R K C D$ & $A L K$ \\
\hline
\end{tabular}

Table 5 Features that distinguish cellular dermatofibroma from dermatofibrosarcoma protuberans

\begin{tabular}{ll}
\hline Cellular dermatofibroma & Dermatofibrosarcoma protuberans \\
\hline $\begin{array}{l}\text { Epidermal hyperplasia } \\
\begin{array}{l}\text { Lateral hyaline collagen } \\
\text { entrapment }\end{array}\end{array}$ & $\begin{array}{l}\text { No epidermal hyperplasia } \\
\text { Diffuse infiltration of dermis }\end{array}$ \\
$\begin{array}{l}\text { Mixed fascicular and storiform } \\
\text { Pale eosinophilic cytoplasm }\end{array}$ & Tight storiform pattern \\
Superficial fat entrapment & Minimal cytoplasm \\
CD34 usually negative & Diffuse infiltration of fat \\
SMA multifocally positive & SMA negative \\
\hline
\end{tabular}

metastases develop [26-28]. The features that distinguish cellular dermatofibroma from dermatofibrosarcoma protuberans are summarized in Table 5. Dermatofibrosarcoma protuberans has a peak incidence in young adults between the second and fourth decades, although children can also be affected; the trunk is the most common anatomic location [25]. Patients with dermatofibrosarcoma protuberans often present when a nodular lesion ("protuberans") develops within a longstanding plaque. Given the high risk for local recurrence, wide surgical margins are advisable. Dermatofibrosarcoma protuberans is diffusely infiltrative with no (or limited) reaction in the overlying epidermis or adjacent dermis (unlike dermatofibroma) (Fig. 5). Dermatofibrosarcoma protuberans shows a characteristic storiform architecture and is composed of bland, hyperchromatic, slender spindle cells with indistinct cytoplasm and remarkably uniform cytomorphology. Infiltration into subcutaneous tissue shows a typical "honeycomb" architecture, often with clusters and linear arrays of adipocytes ("strings of pearls") left within tumor. Nearly all cases of dermatofibrosarcoma protuberans are strongly and diffusely positive for CD34 (see Fig. 5d); this finding is diagnostically useful, despite the low specificity of CD34 (which is also positive in solitary fibrous tumor, spindle cell/pleomorphic lipoma, and soft tissue perineurioma, among other tumor types). Although 5\% of cellular dermatofibromas also express CD34, in those tumors staining is typically only focal or patchy, in contrast to the uniform and strong pattern of staining in dermatofibrosarcoma protuberans. Of note, there is a risk of misdiagnosis of the benign neoplasm cutaneous perineurioma as dermatofibrosarcoma protuberans in a limited biopsy (particularly without knowledge of the clinical appearances of the lesion), given their similar architectural patterns (i.e., both show storiform architecture) and shared CD34 expression; EMA and claudin-1 expression supports perineurioma in this context [29].

As mentioned previously, the fibrosarcomatous variant of dermatofibrosarcoma protuberans is defined as the progression from the conventional storiform growth pattern of slender, hyperchromatic cells to a fascicular, fibrosarcoma-like appearance, with vesicular nuclei and often a high mitotic rate (Fig. 6) [26, 27]. Fibrosarcomatous dermatofibrosarcoma protuberans often shows loss of (or decreased) CD34 expression. Some cases of fibrosarcomatous dermatofibrosarcoma protuberans contain so-called "myoid nodules" (see Fig. 6b). Transformation to the fibrosarcomatous variant is associated with acquisition of metastatic potential (10-15\%), most often to the lungs (see Fig. 6c), as well as more locally destructive growth; external-beam radiation therapy will be considered following resection of fibrosarcomatous dermatofibrosarcoma protuberans in many sarcoma centers. Very rarely, dermatofibrosarcoma protuberans can transform into a pleomorphic (as opposed to a uniform spindle cell) sarcoma [30].

Dermatofibrosarcoma protuberans harbors a characteristic genetic alteration, namely, an unbalanced translocation (often within ring chromosomes): $\operatorname{der}(17)(17 ; 22)(\mathrm{q} 22 ; \mathrm{q} 13)$, resulting in the COL1A1-PDGFB gene rearrangement, which brings the strong collagen type 1 alpha 1 promoter to drive high-level expression of platelet-derived growth factor subunit B [31-33]. Fibrosarcomatous transformation is often associated with increased copies of the fusion gene [34]. FISH for $P D G F B$ can therefore be used to support the diagnosis of dermatofibrosarcoma protuberans [35], although molecular genetic confirmation is rarely needed for conventional dermatofibrosarcoma protuberans; this assay is most useful for fibrosarcomatous examples in which a conventional component is lacking, or for metastatic fibrosarcomatous dermatofibrosarcoma protuberans (see Fig. 6d), when there is incomplete clinical history. Patients with dermatofibrosarcoma protuberans can be 
Fig. 5 Dermatofibrosarcoma protuberans. a. This plaque-like example shows a diffusely infiltrative growth pattern. Unlike dermatofibromas, there is no epidermal hyperplasia. b The honeycomb appearance of the infiltrated subcutaneous fat results in linear collections of adipocytes with a "string of pearls" appearance. c The tumor shows a uniform storiform architecture and contains spindle cells with hyperchromatic nuclei. d Diffuse expression of CD34 is a typical finding
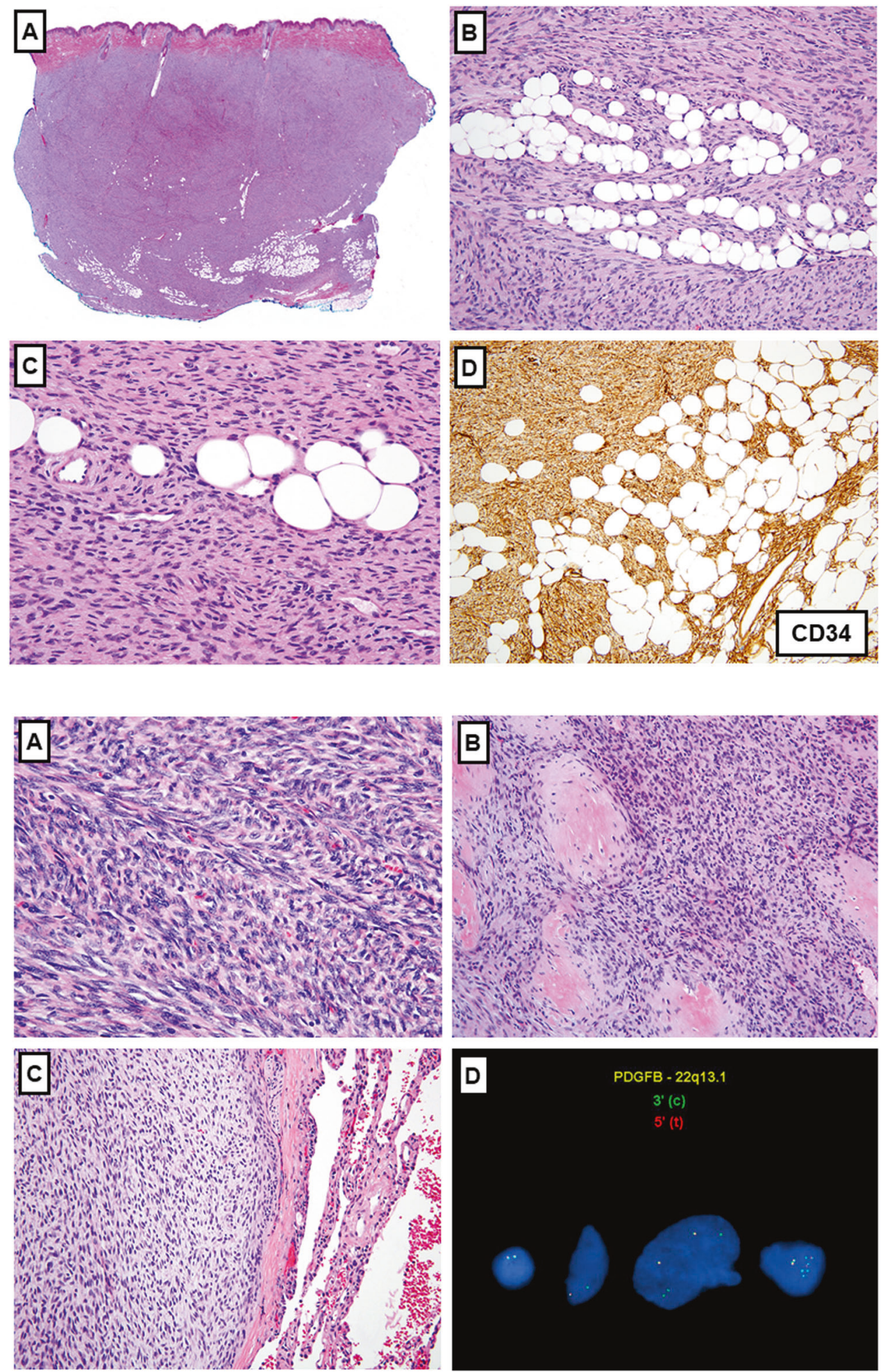

Fig. 6 Fibrosarcomatous dermatofibrosarcoma protuberans. a The tumor shows a fascicular fibrosarcoma-like architecture and contains elongated spindle cells with vesicular chromatin. b "Myoid nodules" are sometimes seen in dermatofibrosarcoma protuberans, most often in the fibrosarcomatous variant. c Lung metastases can be challenging to diagnosis without clinical history. d Fluorescence in situ hybridization (FISH) for $P D G F B$ is a helpful diagnostic adjunct. Note the isolated 3' (centromeric) signals, which are multiple in this case treated by targeted tyrosine kinase inhibitor therapy with agents such as imatinib mesylate, which results in a high rate of partial responses and stable disease, and a low rate of complete responses. Imatinib can be used in the neoadjuvant setting to "downstage" patients with recurrent dermatofibrosarcoma protuberans arising in difficult anatomic locations (such as the axilla or vulva) prior to surgery, in order to avoid significant morbidity [36, 37]. Unfortunately, imatinib is less effective for patients with metastatic fibrosarcomatous dermatofibrosarcoma protuberans. 
Fig. 7 Giant cell fibroblastoma. a The tumor is hypocellular with a haphazard distribution of spindle cells and occasional giant cells in a collagenous stroma. b Dilated pseudovascular ("angiectoid") spaces are characteristic. Note the giant cells
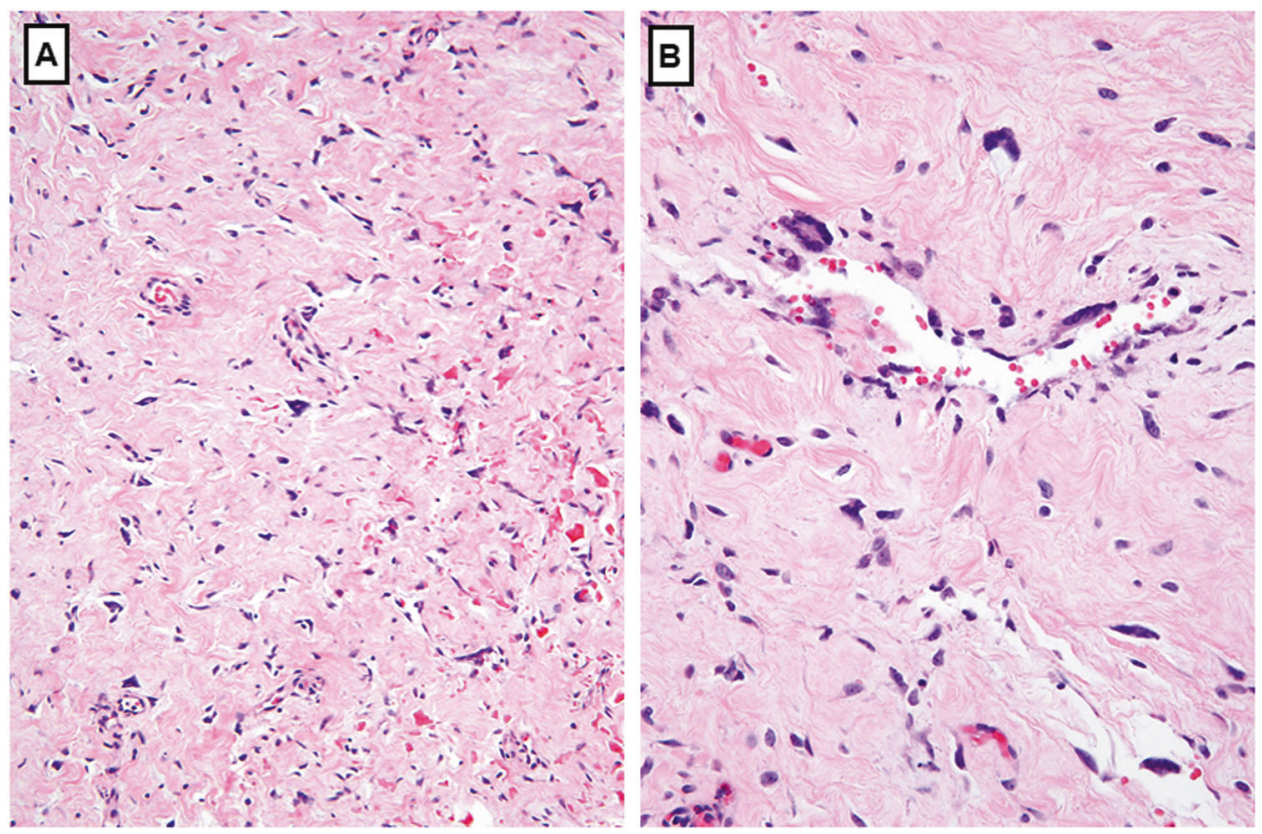

It has been known for some time that $\sim 4 \%$ of cases of dermatofibrosarcoma protuberans are negative for $P D G F B$ rearrangement by FISH. Until recently, the molecular pathogenetic basis for such tumors was unknown. Two recent studies have demonstrated that around half of these cases (2\%) harbor "cryptic" COL1A1$P D G F B$ rearrangements, which can be identified by nextgeneration sequencing; the other $2 \%$ harbor alternate, novel gene fusions: COL6A3-PDGFD, or, more rarely, EMILIN2-PDGFD [38, 39]. Gene expression profiling has indicated that cases of dermatofibrosarcoma protuberans with the alternate PDGFD gene fusions co-cluster with cases that harbor the dominant translocation [39]. It remains to be seen whether patients with dermatofibrosarcoma protuberans with these uncommon fusions will benefit from similar targeted therapies, although it seems likely.

Giant cell fibroblastoma is a rare fibroblastic neoplasm that usually presents in the first decade, shows a male predominance, and has a predilection for the trunk (similar to dermatofibrosarcoma protuberans) [40, 41]. Although giant cell fibroblastoma recurs locally, usually not in the repeated, often destructive fashion as dermatofibrosarcoma protuberans. Remarkably, giant cell fibroblastoma may co-exist with dermatofibrosarcoma protuberans (as hybrid tumors) or recur as dermatofibrosarcoma protuberans. Analogous to dermatofibrosarcoma protuberans, giant cell fibroblastoma harbors a similar $\mathrm{t}(17 ; 22)$ with COL1A1-PDGFB, but often in a balanced translocation with linear (not ring) chromosomes $[33,42]$. Giant cell fibroblastoma is thus essentially a "juvenile" variant of dermatofibrosarcoma protuberans (although as mentioned previously, conventional dermatofibrosarcoma protuberans may also arise during childhood) [41]. Giant cell fibroblastoma is a hypocellular, usually collagen-rich but occasionally myxoid neoplasm consisting of a haphazard arrangement of bland, short spindle cell with hyperchromatic nuclei and occasional multinucleated tumor cells, which characteristically line dilated, pseudovascular ("angiectoid") spaces, a helpful diagnostic clue (Fig. 7). Giant cell fibroblastoma shares infiltrative margins with dermatofibrosarcoma protuberans. Of note, giant cell fibroblastoma does not transform to a fibrosarcomatous, metastasizing variant.

\section{Summary}

"Fibrohistiocytic" is a misnomer; most cutaneous tumors in this category show fibroblastic or myofibroblastic differentiation. Dermatofibroma and variants may harbor gene rearrangements involving $P R K C$, whereas epithelioid fibrous histiocytoma contains $A L K$ gene fusions. The distinction between cellular dermatofibroma and dermatofibrosarcoma protuberans can be made by careful attention to histologic features in most cases. In challenging cases, FISH for $P D G F B$ can be used to confirm the diagnosis of dermatofibrosarcoma protuberans; this is particularly useful for the fibrosarcomatous variant. Unlike conventional dermatofibrosarcoma protuberans, fibrosarcomatous dermatofibrosarcoma protuberans has metastatic potential. Because of the underlying gene rearrangement, dermatofibrosarcoma protuberans can be effectively treated by imatinib in many cases. 


\section{Compliance with ethical standards}

Conflict of interest The author declares that he has no conflict of interest.

Publisher's note Springer Nature remains neutral with regard to jurisdictional claims in published maps and institutional affiliations.

\section{References}

1. Ozzello L, Stout AP, Murray MR. Cultural characteristics of malignant histiocytomas and fibrous xanthomas. Cancer. 1963; 16:331-44.

2. Fletcher CD. Pleomorphic malignant fibrous histiocytoma: fact or fiction? A critical reappraisal based on 159 tumors diagnosed as pleomorphic sarcoma. Am J Surg Pathol. 1992;16:213-28.

3. Fletcher CD, Gustafson P, Rydholm A, et al. Clinicopathologic reevaluation of 100 malignant fibrous histiocytomas: prognostic relevance of subclassification. J Clin Oncol. 2001;19:3045-50.

4. Hornick JL. Subclassification of pleomorphic sarcomas: How and why should we care? Ann Diagn Pathol. 2018;37:118-24.

5. Elder D, Massi D, Scolyer R, Willemze R. WHO Classification of Skin Tumours. Lyon: International Agency for Research on Cancer; 2018.

6. Calonje E, Mentzel T, Fletcher CD. Cellular benign fibrous histiocytoma. Clinicopathologic analysis of 74 cases of a distinctive variant of cutaneous fibrous histiocytoma with frequent recurrence. Am J Surg Pathol. 1994;18:668-76.

7. Volpicelli ER, Fletcher CD. Desmin and CD34 positivity in cellular fibrous histiocytoma: an immunohistochemical analysis of 100 cases. J Cutan Pathol. 2012;39:747-52.

8. Calonje E, Fletcher CD. Aneurysmal benign fibrous histiocytoma: clinicopathological analysis of 40 cases of a tumour frequently misdiagnosed as a vascular neoplasm. Histopathology. $1995 ; 26: 323-31$.

9. Enzinger FM. Angiomatoid malignant fibrous histiocytoma: a distinct fibrohistiocytic tumor of children and young adults simulating a vascular neoplasm. Cancer. 1979;44:2147-57.

10. Costa MJ, Weiss SW. Angiomatoid malignant fibrous histiocytoma. A follow-up study of 108 cases with evaluation of possible histologic predictors of outcome. Am J Surg Pathol. 1990;14:1126-32.

11. Fanburg-Smith JC, Miettinen M. Angiomatoid "malignant" fibrous histiocytoma: a clinicopathologic study of 158 cases and further exploration of the myoid phenotype. Hum Pathol. 1999;30:1336-43.

12. Rossi S, Szuhai K, Ijszenga M, et al. EWSR1-CREB1 and EWSR1-ATF1 fusion genes in angiomatoid fibrous histiocytoma. Clin Cancer Res. 2007;13:7322-8.

13. Antonescu CR, Dal Cin P, Nafa K, et al. EWSR1-CREB1 is the predominant gene fusion in angiomatoid fibrous histiocytoma. Genes Chromosomes Cancer. 2007;46:1051-60.

14. Kaddu S, McMenamin ME, Fletcher CD. Atypical fibrous histiocytoma of the skin: clinicopathologic analysis of 59 cases with evidence of infrequent metastasis. Am J Surg Pathol. 2002;26:35-46.

15. Płaszczyca A, Nilsson J, Magnusson L, et al. Fusions involving protein kinase $\mathrm{C}$ and membrane-associated proteins in benign fibrous histiocytoma. Int J Biochem Cell Biol. 2014;53:475-81.

16. Walther C, Hofvander J, Nilsson J, et al. Gene fusion detection in formalin-fixed paraffin-embedded benign fibrous histiocytomas using fluorescence in situ hybridization and RNA sequencing. Lab Invest. 2015;95:1071-6.

17. Jones EW, Cerio R, Smith NP. Epithelioid cell histiocytoma: a new entity. Br J Dermatol. 1989;120:185-95.
18. Glusac EJ, Barr RJ, Everett MA, et al. Epithelioid cell histiocytoma. A report of 10 cases including a new cellular variant. Am J Surg Pathol. 1994;18:583-90.

19. Singh Gomez C, Calonje E, Fletcher CD. Epithelioid benign fibrous histiocytoma of skin: clinico-pathological analysis of 20 cases of a poorly known variant. Histopathology. 1994;24:123-9.

20. Doyle LA, Fletcher CD. EMA positivity in epithelioid fibrous histiocytoma: a potential diagnostic pitfall. J Cutan Pathol. 2011;38:697-703.

21. Jedrych J, Nikiforova M, Kennedy TF, et al. Epithelioid cell histiocytoma of the skin with clonal ALK gene rearrangement resulting in VCL-ALK and SQSTM1-ALK gene fusions. Br J Dermatol. 2015;172:1427-9.

22. Doyle LA, Mariño-Enriquez A, Fletcher CD, et al. ALK rearrangement and overexpression in epithelioid fibrous histiocytoma. Mod Pathol. 2015;28:904-12.

23. Dickson BC, Swanson D, Charames GS, et al. Epithelioid fibrous histiocytoma: molecular characterization of ALK fusion partners in 23 cases. Mod Pathol. 2018;31:753-62.

24. Kazakov DV, Kyrpychova L, Martinek P, et al. ALK gene fusions in epithelioid fibrous histiocytoma: a study of 14 cases, with new histopathological findings. Am J Dermatopathol. 2018;40:805-14.

25. Connelly JH, Evans HL. Dermatofibrosarcoma protuberans. A clinicopathologic review with emphasis on fibrosarcomatous areas. Am J Surg Pathol. 1992;16:921-5.

26. Mentzel T, Beham A, Katenkamp D, et al. Fibrosarcomatous ("high-grade") dermatofibrosarcoma protuberans: clinicopathologic and immunohistochemical study of a series of 41 cases with emphasis on prognostic significance. Am J Surg Pathol. 1998;22:576-87.

27. Goldblum JR, Reith JD, Weiss SW. Sarcomas arising in dermatofibrosarcoma protuberans: a reappraisal of biologic behavior in eighteen cases treated by wide local excision with extended clinical follow up. Am J Surg Pathol. 2000;24:1125-30.

28. Abbott JJ, Oliveira AM, Nascimento AG. The prognostic significance of fibrosarcomatous transformation in dermatofibrosarcoma protuberans. Am J Surg Pathol. 2006;30:436-43.

29. Hornick JL, Fletcher CD. Soft tissue perineurioma: clinicopathologic analysis of 81 cases including those with atypical histologic features. Am J Surg Pathol. 2005;29:845-58.

30. Swaby MG, Evans HL, Fletcher CD, et al. Dermatofibrosarcoma protuberans with unusual sarcomatous transformation: a series of 4 cases with molecular confirmation. Am J Dermatopathol. 2011;33:354-60.

31. Pedeutour F, Simon MP, Minoletti F, et al. Ring 22 chromosomes in dermatofibrosarcoma protuberans are low-level amplifiers of chromosome 17 and 22 sequences. Cancer Res. 1995;55:2400-3.

32. Minoletti F, Miozzo M, Pedeutour F, et al. Involvement of chromosomes 17 and 22 in dermatofibrosarcoma protuberans. Genes Chromosomes Cancer. 1995;13:62-5.

33. Simon MP, Pedeutour F, Sirvent N, et al. Deregulation of the platelet-derived growth factor B-chain gene via fusion with collagen gene COL1A1 in dermatofibrosarcoma protuberans and giant-cell fibroblastoma. Nat Genet. 1997;15:95-8.

34. Abbott JJ, Erickson-Johnson M, Wang X, et al. Gains of COL1A1-PDGFB genomic copies occur in fibrosarcomatous transformation of dermatofibrosarcoma protuberans. Mod Pathol. 2006;19:1512-8.

35. Karanian M, Pérot G, Coindre JM, et al. Fluorescence in situ hybridization analysis is a helpful test for the diagnosis of dermatofibrosarcoma protuberans. Mod Pathol. 2015;28:230-7.

36. Rutkowski $P$, Van Glabbeke $M$, Rankin $\mathrm{CJ}$, et al. Imatinib mesylate in advanced dermatofibrosarcoma protuberans: pooled analysis of two phase II clinical trials. J Clin Oncol. 2010;28:1772-9. 
37. Stacchiotti S, Pantaleo MA, Negri T, et al. Efficacy and Biological Activity of Imatinib in Metastatic Dermatofibrosarcoma Protuberans (DFSP). Clin Cancer Res. 2016;22:837-46.

38. Dadone-Montaudié B, Alberti L, Duc A, et al. Alternative PDGFD rearrangements in dermatofibrosarcomas protuberans without PDGFB fusions. Mod Pathol. 2018;31:1683-93.

39. Dickson BC, Hornick JL, Fletcher CDM, et al. Dermatofibrosarcoma protuberans with a novel COL6A3-PDGFD fusion gene and apparent predilection for breast. Genes Chromosomes Cancer. 2018;57:437-45.
40. Dymock RB, Allen PW, Stirling JW, et al. Giant cell fibroblastoma. A distinctive, recurrent tumor of childhood. Am J Surg Pathol. 1987;11:263-71.

41. Shmookler BM, Enzinger FM, Weiss SW. Giant cell fibroblastoma. A juvenile form of dermatofibrosarcoma protuberans. Cancer. 1989;64:2154-61.

42. Dal Cin P, Sciot R, de Wever I, et al. Cytogenetic and immunohistochemical evidence that giant cell fibroblastoma is related to dermatofibrosarcoma protuberans. Genes Chromosomes Cancer. 1996;15:73-5. 